\title{
Factors Affecting the Design of Untethered Magnetic Haptic Interfaces
}

\author{
Joseph B. Brink* $\quad$ Andrew J. Petruska ${ }^{\dagger} \quad$ David E. Johnson $\quad$ Jake J. Abbott ${ }^{\S}$
}

University of Utah

\begin{abstract}
This paper presents an analysis of the factors affecting the performance, workspace, and stability of untethered magnetic haptic interfaces, with the goal of informing the design of future devices. We differentiate untethered magnetic interfaces, which use a stylus with an embedded permanent magnet to interact with magnetic fields projected into space by electromagnets, from the more wellknown Lorentz-force magnetic interfaces, which work on different principles. We show that even though untethered magnetic interfaces have little to no damping, they still exhibit inherent stability properties as part of a sampled-data system. Although a ferromagnetic core often enables increased forces to be rendered at distances farther from the electromagnet, we show that there are cases in which larger forces can be rendered and cases in which stiffer virtual walls can be rendered by removing the ferromagnetic core.
\end{abstract}

Index Terms: H.5.2 [Information Interfaces and Presentation]: User Interfaces-Haptic I/O

\section{INTRODUCTION}

This paper presents an analysis of the factors affecting the performance, workspace, and stability of untethered magnetic haptic interfaces. Magnetic haptic interfaces differ from traditional kinesthetic haptic displays, which use one or more back-drivable DC motors in a kinematic chain to display forces to a user's hand. No matter how well designed, there will always be some inertia and friction from this small robotic device that will impact the haptic experience of the user. The practical consequence is that it is difficult to accurately render very low friction or very low inertia environments and tools. Some applications, such as microsurgical training, require device performance specifications beyond what is currently possible with traditional haptics interfaces. Magnetic haptic interfaces have received significant attention in the last decade, in part due to their potential to render low-friction environments, as they enable force transmission without a kinematic chain.

We can classify magnetic haptic interfaces considered to date into two distinct technologies: Lorentz-force interfaces and untethered interfaces. The most well-known magnetic haptic interface is probably the Butterfly Haptics Maglev haptic interface, which was originally developed by Berkelman and Hollis [5]. This is a Lorentz-force interface. An earlier example is that of Salcudean and Vlaar [17]. Lorentz-force interfaces are similar to DC motors in their method of transducing electrical current into force/torque. If a wire passes in the region between two strong magnets, then forces will be generated on the wire, with a force magnitude that is linear with current flowing through the wire and a force direction that is always orthogonal to both the wire and the field. If multiple Lorentz-force actuators of this type apply forces at different locations on a stylus, then full six-degree-of-freedom (6-DOF) haptic

\footnotetext{
*e-mail: joey.brink@utah.edu

†email: andrew.petruska@utah.edu

‡e-mail: dejohnso@cs.utah.edu

§e-mail: jake.abbott@utah.edu
}

IEEE Haptics Symposium 2014

23-26 February, Houston, Tx, USA

978-1-4799-3130-9/14/\$31.00 @2014 IEEE

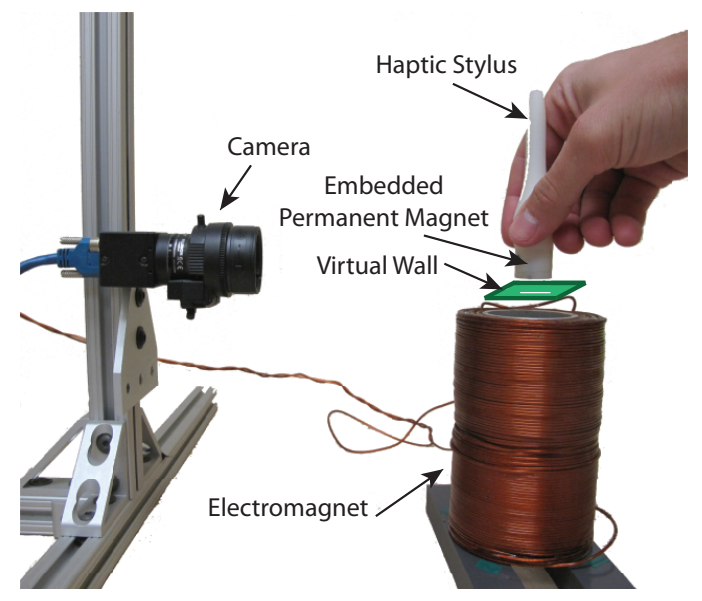

Figure 1: In an untethered magnetic haptic interface, a virtual wall is rendered in space by an electromagnetic system, and a fully untethered stylus with an embedded permanent magnet interacts with the virtual wall. An untethered stylus requires some form of external tracking; here, we use a camera with a controlled region-of-interest.

rendering is possible. Lorentz-force interfaces are able to generate large crisp forces with a frictionless stylus, but the nature of their construction is such that the stylus will likely have a relatively large inertia, and the motion range of the stylus will be limited due to the need to keep the current-carrying wires within their respective magnetic fields. For example, the Maglev has a stylus inertia of $616 \mathrm{~g}$, and workspace limitations of $\pm 8^{\circ}$ in orientation about each axis through a 24-mm-diameter spherical workspace [5]. Berkelman and Dzadovsky have extended the workspace of the Maglev with a novel coil shape and magnet configuration to achieve a workspace of $\pm 30^{\circ}$ in orientation about each axis through a 50-mm-diameter sphere [4].

In this paper, we are not concerned with Lorentz-force interfaces, but rather, untethered interfaces, which are fundamentally different in their operation. An untethered interface works by rendering forces to a permanent magnet attached to a stylus via electromagnets that project their magnetic fields into space (Fig. 1). The field shape of an electromagnet cannot be changed, but the magnitude of the field at each location in space is linear with the current flowing through the electromagnet, and this current can be controlled in real-time. The position, and possibly orientation, of the permanent magnet must be tracked in space to provide feedback for the device to work as a haptic interface. This tracking problem is not trivial for untethered interfaces. Although at the most fundamental level, untethered interfaces also capitalize on the Lorentz force, they do not utilize the type of Lorentz-force actuators that have come to characterize these interfaces. Instead, the stylus is truly untethered and can be completely removed from the electromagnet system.

Untethered interfaces are desirable in that, rather that using a generic stylus, the actual tool being simulated can be used as the stylus for increased realism; the tool must simply be modified with a permanent magnet at the point where forces are to be rendered. 
Untethered interfaces are also desirable in that they do not have the same range-of-motion limitations inherent to Lorentz-force interfaces; there is fundamentally nothing to prohibit the stylus from being held at any orientation. Work from the magnetic-manipulation community has shown that real-time control over force (3-DOF) and torque (2-DOF) on a magnetic device is possible with the device in any orientation, provided enough electromagnets are used in the right configuration [16]. Note that with the untethered stylus described above, it will not be possible to generate torque about the dipole moment of the stylus magnet (e.g., the roll direction of the stylus); this is a limitation of untethered magnetic interfaces.

There are a number of prior examples of untethered magnetic interfaces. Hu et al. [15] proposed an open-surgery simulator comprising an untethered permanent-magnet surgical tool to be actuated by a hemispherical array of electromagnets. Preliminary experiments with a 1-DOF test platform generated a maximum force of $8 \mathrm{~N}$. However, it is not clear if the proposed concept has ever been brought to reality. Staas de Jong developed the Cyclotactor: a 1-DOF untethered magnetic interface in which the user wears a magnetic thimble on the end of one finger, which is levitating above an electromagnet $[8,9]$. Infrared sensing techniques provide a 0.2 $\mathrm{mm}$ height resolution through a vertical workspace of $35 \mathrm{~mm}$, with a sampling rate of $625 \mathrm{~Hz}$. The interface can render virtual surfaces and high-frequency vibrations. To eliminate constraints on the horizontal workspace found in the Lorentz-force devices, Berkelman and Dzadovsky have developed a planar array of cylindrical electromagnetic coils that work together to apply 6-DOF force/torque to a levitating stylus located above the planar array. Force/torque is applied to multiple independent magnets on the stylus, reminiscent of a Maglev device stylus. With the original 10 coils, the horizontal workspace for haptic interaction was $80 \times 60 \mathrm{~mm}$ [4], and they have since added 17 more coils, further increasing the horizontal workspace [3]. To date, the work on untethered magnetic interfaces has largely consisted of novel designs, device characterization, and proof-of-concept experiments. This field does not yet have a cohesive framework to describe the performance of various devices.

For a decade from the mid-1990s to the mid-2000s, substantial work was done to characterize the behavior of traditional haptic interfaces implementing virtual spring and spring-damper walls $[7,11,2,1,10]$. Assumptions started simple, treating the haptic interface as a 1-DOF mass-damper with a controllable applied force, being controlled as a sampled-data system. As the field progressed, others began to consider the effects of encoder quantization and nonlinear friction in their analysis. Some works were interested in stability, which is concerned with the response of signals in closedloop systems, and some were interested in passivity, which is concerned with the energy flow from one system to another, with an understanding that passivity is a sufficient but not necessary condition for stable interaction. The importance of the zero-order-hold effect of discrete sampling was considered early on, but the electrical time constant of the motor has typically been assumed to be very fast and negligible, such that the applied force is assumed to be constant between updates.

It is our belief that researchers working in the field of untethered magnetic interfaces must retrace the steps taken with traditional haptic interfaces. We do not yet have a good understanding of how different parameters - such as inertia, sampling rate, sensor quantization, and inductance-affect the stability/passivity, the size/condition of the workspace, and the achievable force range for an untethered interface. Although it will not use optical encoders like a traditional interface, the position-feedback method for an untethered interface may still include quantization effects. For air-core electromagnets, we should assume no form of friction/damping in the interface, but for ferromagnetic-core electromagnets, we should acknowledge the effect of eddy-current damping between the permanent magnet and the core [12]. Unlike tra- ditional interfaces, it is probably incorrect to assume that electrical time constants are negligible, since the inductance of the electromagnets is likely to be large, which complicates the zero-order-hold effect. Finally, in possibly the largest divergence from traditional interfaces, the magnetic field established between samples acts as a continuous nonlinear spring, such that the force rendered to the user varies continuously with the position of the stylus between samples.

Inspired by the Colgate and Brown [6] and Gillespie and Cutkosky [11] works on 1-DOF traditional haptic interfaces, we present this paper to provide preliminary analysis, simulations, and experimental evidence for a number of factors that affect the design of a 1-DOF untethered magnetic interface. We show that even though untethered magnetic interfaces have little to no damping, they still exhibit inherent stability properties in a sampled-data system. Although a ferromagnetic core often enables increased forces to be rendered at distances farther from the electromagnet, we show that there are cases in which larger forces can be rendered without the ferromagnetic core. Finally, we build and report on workspace characterization of a 1-DOF untethered magnetic interface. We conduct an experiment that demonstrates that stiffer virtual walls can be rendered with the air core than with a ferromagnetic core, and that stability is insensitive to sampling rate, both of which are counterintuitive. Although we do not feel that any of the topics that we cover in this paper are fully resolved, our goal here is to provide a set of insights about untethered magnetic interfaces that can be used to inform the design of future devices, and which can be used as a jumping-off point for future investigation.

Throughout this paper, we will use the following convention: scalar variables are represented with standard font, irrespective of upper/lower case; vectors are represented with bold font, irrespective of upper/lower case; and matrices are represented with blackboard-bold font.

\section{Stability and Energetic Properties}

The fundamental difference between traditional and magnetic virtual walls lies in the way that intended force is actually rendered by the haptic display in a sampled-data sense. In a traditional virtual wall, throughout each sampling period $T$ the force rendered to the stylus is typically assumed to remain constant (Fig. 2(a)), which can be quite accurate if the electrical time constant is fast. In contrast, a magnetic virtual wall establishes a magnetic field and has its own non-negligible dynamics due to inductance, and more fundamentally, throughout one sampling period $T$ the force rendered to the stylus by the (potentially varying) magnetic field varies continuously with the position of the stylus (Fig. 2(b)).

To begin to explore how the stability of a magnetic virtual wall differs from that of a traditional virtual wall, we construct a "bouncing ball" contact model inspired by [11], shown in Fig. 3. The human hand/finger is modeled as a mass-spring-damper system that is rigidly attached to the stylus, which is modeled as a mass-damper system. The stylus can make and break contact with the wall, which is implemented as a linear spring with a unilateral constraint (that is, $F_{w}=K_{w} y$ when the stylus is inside the wall, and $F_{w}=0$ otherwise). The dynamic model is described by

$$
\left(m_{s}+m_{h}\right) \ddot{y}+\left(b_{s}+b_{h}\right) \dot{y}+k_{h} y=F_{g}+F_{h}-F_{w}
$$

where $F_{g}$ is the force of gravity (i.e., the weight) and $F_{h}$ is the force from the hand on the stylus, both of which are positive when pushing down, and $F_{w}$ is the force applied by the wall, which is positive when pushing up. In practice, the virtual wall force $F_{w}$ will deviate from the intended relationship due to the effects shown in Fig. 2.

To simplify our analysis, we assume the first-order approximation for the magnetic field produced by the electromagnet: the dipole-field. Although the dipole field is an approximation, we believe it will provide insight into the stability of magnetic virtual walls in general because it captures the fundamental nonlinearities 
(a)

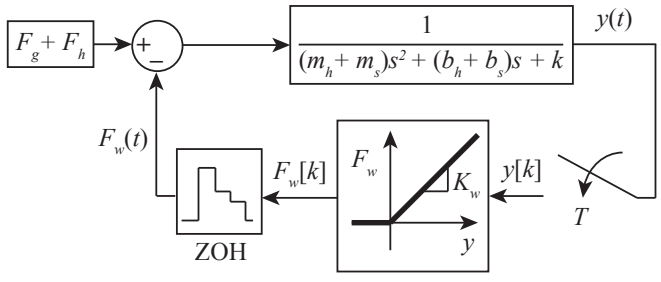

(b)

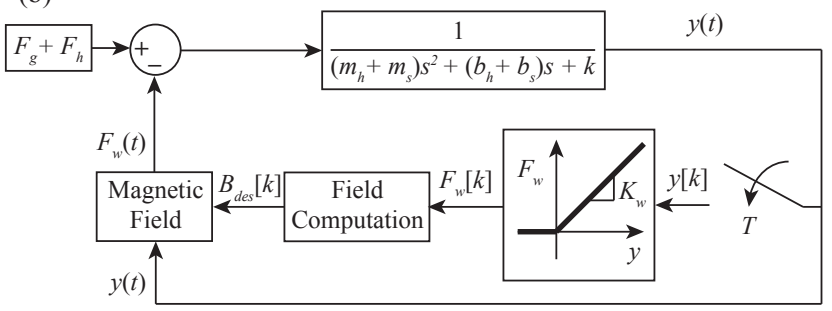

Figure 2: (a) Rendering a traditional virtual wall as a sampled-data system. (b) Rendering a magnetic virtual wall as a sampled-data system, with continuously varying magnetic forces between updates. The hand/finger model used is that of Fig. 3 (our mix of frequency and time domain is done for simplicity). In both systems, the wall is rendered as a linear spring $F_{w}=K_{w} y$ with a unilateral constraint.

in the magnetic field. Similarly, we also model the magnet in the stylus as a dipole-field source. Assuming that the stylus magnet is located directly above the electromagnet (i.e., along the dipole axis), and that the dipole of the stylus magnet is parallel and opposing the electromagnet's dipole, we can model the repulsive force applied to the stylus simply as the force between two dipoles, $F_{\mathrm{dd}}$ :

$$
F_{\mathrm{dd}}=\frac{3 \mu_{0} m_{\mathrm{sty}} m_{\mathrm{elec}}}{4 \pi p^{4}}
$$

where $m_{\text {sty }}$ and $m_{\text {elec }}$ are the strength of the stylus and electromagnet dipoles, respectively, in units $\mathrm{A} \cdot \mathrm{m}^{2}, p$ represents the distance between the dipoles in units of meters, $\mu_{0}$ is the permeability of free space, and the dipole-dipole force is in units of newtons.

Our goal is to establish a virtual wall with spring constant $K_{w}$ at some distance $y_{w}$ from the dipole. At a given sample $k$, we set the current through the electromagnet based on the position $y[k]$. The electromagnet's dipole $m_{\text {elec }}$ is a linear function of this current through a known mapping (i.e., the unit-current dipole strength). We choose $m_{\text {elec }}$, and thus the current, to set the force of the magnetic virtual wall to be equal to the linear wall that we are trying to render:

$$
F_{w}[k]=\frac{3 \mu_{0} m_{\text {sty }} m_{\text {elec }}}{4 \pi\left(y_{w}-y[k]\right)^{4}}=K_{w} y[k]
$$

Thus, we set the electromagnet dipole as:

$$
m_{\mathrm{elec}}[k]=\frac{4 \pi K_{w} y[k]\left(y_{w}-y[k]\right)^{4}}{3 \mu_{0} m_{\text {sty }}}
$$

In a traditional virtual wall, the force would be held constant between samples, but with the magnetic virtual wall the force will change between updates due to the movement of the stylus through the magnetic field. The continuous-time position of the stylus will cause the applied force to vary continuously as:

$$
F_{w}(t)=\frac{3 \mu_{0} m_{\text {sty }} m_{\mathrm{elec}}}{4 \pi\left(y_{w}-y(t)\right)^{4}}=\left(\frac{\left(y_{w}-y[k]\right)^{4}}{\left(y_{w}-y(t)\right)^{4}}\right) K_{w} y[k]
$$

where the electromagnet's dipole is assumed to be constant between updates as computed in (4). We will later relax this assumption.

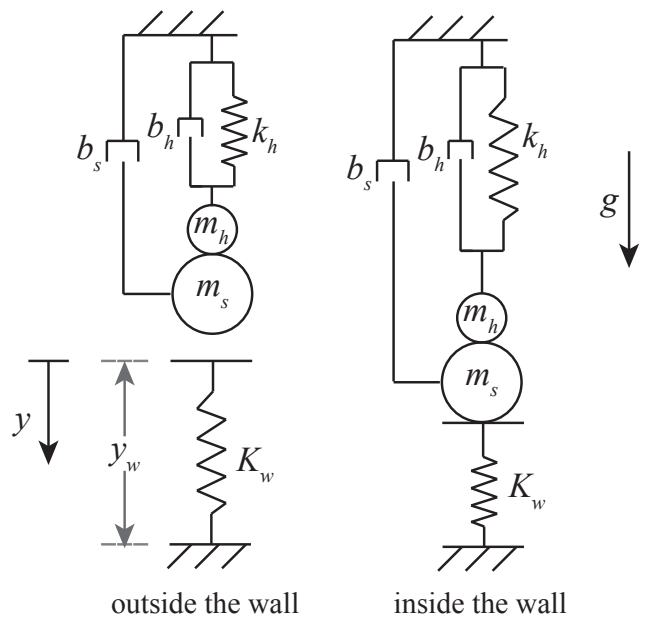

Figure 3: Simple model of the hand-stylus interaction with the virtual wall, based on [11]. The hand (or finger) is modeled as a massspring-damper with parameters $m_{h}, k_{h}, b_{h}$. The stylus is modeled as a mass-damper with parameters $m_{s}, b_{s}$. The stylus is not in contact with the wall for positions $y<0$, and is represented as a simple linear compression spring with spring constant $K_{w}$ for positions $y \geq 0$.

To compare the ideal wall, the traditional virtual wall, and the magnetic virtual wall, we use the following parameters: $m_{h}=$ $0.006 \mathrm{~kg}$, the same value in [11], and consistent with the measured finger impedance in [14] when $\sim 2 \mathrm{~N}$ is applied; $b_{h}=0$, to study stability without damping, as in [11]; $k_{h}=25 \mathrm{~N} / \mathrm{m}$, from [14] when the finger applies $\sim 2 \mathrm{~N}$ of force; $m_{s}=0.020 \mathrm{~kg}$, the approximate weight of the stylus we designed (described later); $b_{s}=0$, to study stability without damping, as in [11]; $F_{g}+F_{h}=2.5 \mathrm{~N}$, corresponding to approximately $2 \mathrm{~N}$ of force intentionally applied by the human and the rest from gravity; $T=0.01 \mathrm{~s}$, the same value in [11]; and $K_{w}=100 \mathrm{~N} / \mathrm{m}$, a much softer wall than the $K_{w}=5000 \mathrm{~N} / \mathrm{m}$ in [11]. We simulate two different magnetic virtual walls: one with the wall placed at $y_{w}=40 \mathrm{~mm}$, and one at $y_{w}=160 \mathrm{~mm}$. In both simulations, the electromagnet is placed below the wall surface located at $y=0$ by the specified amount. The simulation starts with the stylus at $y=0$ and with some initial upward velocity $\dot{y}>0$. Figure 4 shows the resulting interacting with the ideal wall, the traditional virtual wall, and two magnetic virtual walls.

As expected, the ideal virtual wall with no damping results in a bouncing stylus that neither decays away nor grows larger. The traditional virtual wall results in a bouncing stylus that bounces with growing amplitude, which demonstrates the wall's instability. The two magnetic virtual walls result in opposite behavior: the interaction with the magnetic virtual wall with $y_{w}=40 \mathrm{~mm}$ actually decays away and is stable, whereas the interaction with the magnetic virtual wall with $y_{w}=160 \mathrm{~mm}$ behaves more like a traditional virtual wall and grows without bound to instability. In the limit as $y_{w}$ becomes very large, we find that $F_{w}(t)$ behaves more like the force of a traditional wall, in that the force becomes less sensitive to small movements in the stylus due to the shape of the magnetic field far from the dipole. Under these modeling assumptions (i.e., the electromagnet is a dipole, no damping), the magnetic virtual wall will always result in a less unstable interaction than the traditional virtual wall, and sometimes a stable interaction.

The instability that results is due to the well-known "energy leak" in the virtual wall. Due to the discrete updates, it is too easy to penetrate into the virtual wall (compared to the ideal wall), and the wall pushes too hard when the stylus is being removed, with the result being that the virtual wall generates a net amount of energy 


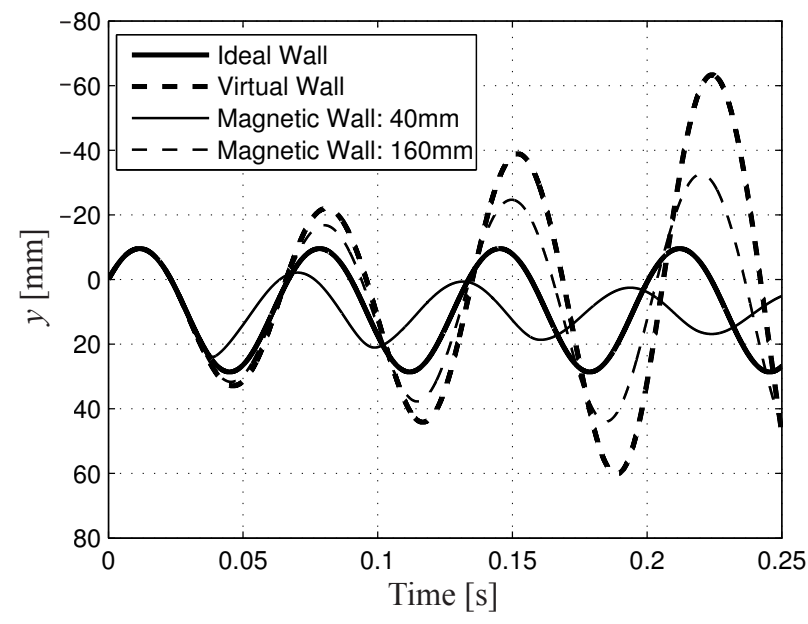

Figure 4: Simulation of a stylus bouncing on an ideal wall, a traditional virtual wall, a magnetic virtual wall rendered $40 \mathrm{~mm}$ away from the electromagnet dipole, and a magnetic virtual wall rendered $160 \mathrm{~mm}$ away from the electromagnet dipole. The ideal wall is marginally stable as expected. The traditional virtual wall and one magnetic virtual wall are unstable, as would be expected. The magnetic virtual wall at $y_{w}=40 \mathrm{~mm}$ actually decays away and is stable, even though no damping is modeled.

for each in-out interaction. However, Fig. 4 implies that there may not be an energy leak on certain magnetic virtual walls.

To further explore energy exchange, we consider an interaction in which a stylus is moved into the wall at a constant speed and then moved out of the wall at the same speed. The work done by the user/stylus to move into the wall, and the work done by the wall on the user/stylus to move out of the wall, are calculated as the force integrated over distance. In Fig. 5, we see that certain magnetic virtual walls (e.g., the wall at $y_{w}=40 \mathrm{~mm}$ ) are actually more dissipative than the ideal wall that they are trying to emulate, and other magnetic virtual walls (e.g., the wall at $y_{w}=160 \mathrm{~mm}$ ) have an energy leak, but are less energetic than a traditional virtual wall of the same stiffness. It appears from these simulations that the critical factor to consider is whether the local stiffness (i.e., the linearization at the location of the stylus) of the magnetic field is more or less stiff than the intended stiffness $K_{w}$ of the virtual wall. If the magnetic field's local stiffness is greater than that of the intended stiffness, we actually see a reversal of the energy-leak phenomenon, with discrete updates leading to a more dissipative interaction.

A factor that we have neglected until now is the non-negligible electrical time constant of real electromagnets; we cannot instantaneously change the current, and thus cannot instantaneously change the magnetic field. Even when running amplifiers in a currentcontrol mode, the maximum rate of change in current (i.e., the slew rate) of an electromagnet depends on the maximum available amplifier voltage $V_{\max }$ and the inductance of the magnet $L$ : $(d I / d t)_{\max }=V_{\max } / L$. We ran new simulations in which the slew rate was accounted for, and the results are shown in Fig. 6. We observe that the slew rate seems to have a kind of amplifying effect on energetic behavior that was observed before considering the slew rate, making it even more dissipative or even more energetic.

\section{Modeling a Magnetic Interface With/Without a FERROMAGNETIC CORE}

In this section we develop an analytical model to enable fast analysis and design optimization without the use of FEA. The model that we will consider here is described in Fig. 7. To determine the
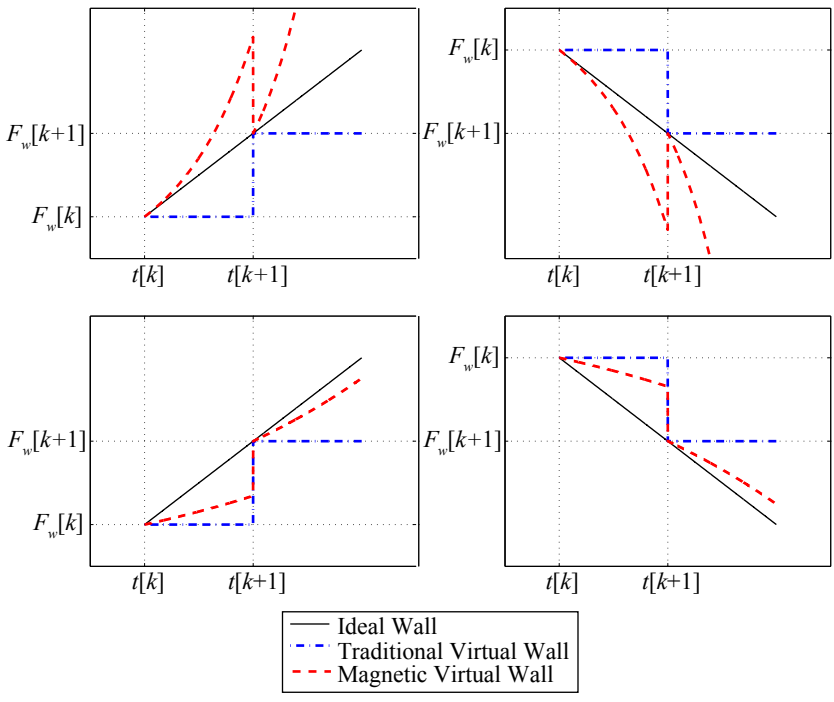

Figure 5: Example force curves for an ideal spring wall, a traditional virtual wall, and a magnetic virtual wall, all with the same intended spring constant. The left column shows movement into the wall, and the right column shows movement out of (but not leaving) the wall. In all cases, the movement is at a constant speed, such that time and distance are proportional. The work done by the wall is proportional to the area under the force curve. A sufficient condition for stable interactions would be that during movement into the wall the area under the force curve is larger than the area under the ideal wall (the ideal wall is neither energetic nor dissipative), and during movement out of the wall the area under the force curve is smaller than the area under the ideal wall. On the top row, we show a magnetic virtual wall that is more dissipative than an ideal wall, and thus stable. On the bottom row, we show a magnetic virtual wall that has an energy leak, but is still less energetic than the traditional virtual wall. In both cases, the traditional virtual wall has an energy leak.
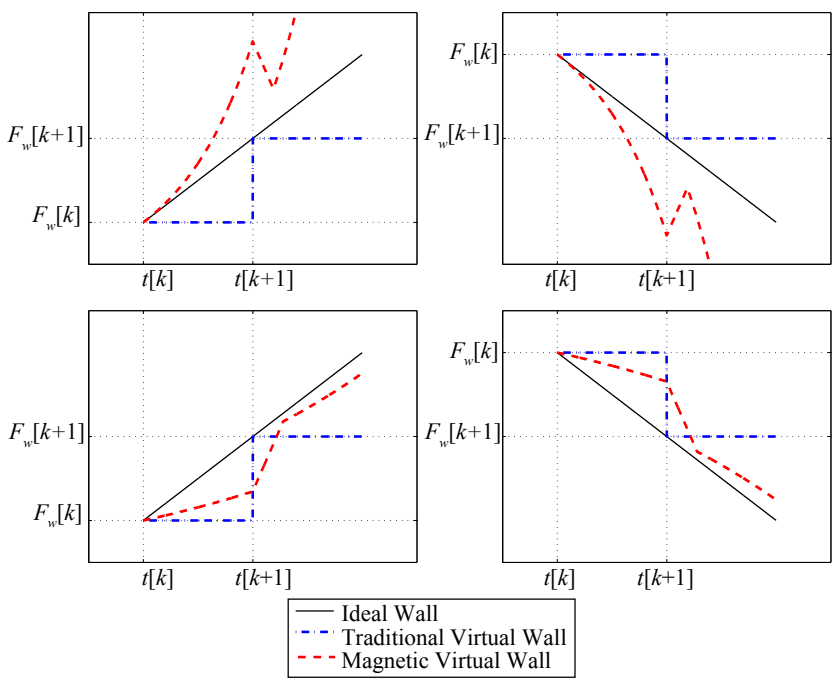

Figure 6: Same experiment shown in Figure 5, but with non-negligible slew rate of the electromagnet. The slew rate appears to amplify the effect of the underlying energy leak: if the interaction was already dissipative, the inclusion of slew rate renders the interaction more dissipative, and if the interaction already had an energy leak, the inclusion of the slew rate renders the interaction more energetic. 


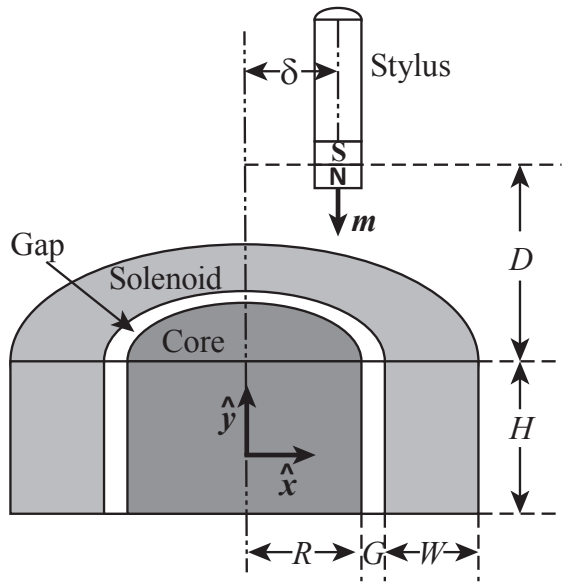

Figure 7: Magnetic interface model. A cylindrical current-carrying solenoid with inner radius $R+G$, thickness $W$, and height $H$ is wrapped around a ferromagnetic core or an air core of radius $R$ and height $H$ with a gap $G$ between the solenoid and the core. An untethered stylus exists within the magnetic field produced by the electromagnet. A permanent magnet, modeled as a point dipole, is located at the end of the stylus. The stylus dipole has height $D$ above the surface of the electromagnet (or height $D+H / 2$ above the origin, which represents the location of the electromagnet's dipole), and an offset $\delta$ from the $\hat{y}$ axis.

force vector $\mathbf{F}$ exerted on the stylus dipole by the electromagnet, we decompose the problem into two distinct parts: determine the force exerted on the stylus due to the solenoid (i.e., the current-carrying wire), and determine the force exerted on the stylus due to the presence of a ferromagnetic core. The total force on the stylus is then the sum of these two solutions, $\mathbf{F}=\mathbf{F}_{\text {sol }}+\mathbf{F}_{\text {core }}$.

\subsection{Force due to the Solenoid}

The force exerted on the stylus dipole due to the solenoid is equal and opposite to the force exerted on the solenoid due to the stylus dipole. This fact allows us to calculate the dipole's magnetic field $\mathbf{B}_{\text {sty }}(\mathbf{p})$ at each differential volume element inside the solenoid, then compute the magnetic force on the differential volume of current flowing through the solenoid:

$$
\mathbf{B}_{\text {sty }}(\mathbf{p})=\frac{\mu_{0}}{4 \pi|\mathbf{p}|^{2}}\left[\frac{3}{|\mathbf{p}|^{2}} \mathbf{p} \mathbf{p}^{T}-\mathbb{I}_{3 \times 3}\right] \mathbf{m}_{\text {sty }}
$$

where $\mathbf{p}$ is the vector from the center of the dipole to each differential volume inside the solenoid. If we apply a current $I$ through the solenoid's wire (with wire cross-sectional area $A$ ), the current density vector through the solenoid will be

$$
\mathbf{J}_{\mathrm{sol}}=\frac{I}{A}\left[\begin{array}{c}
-\sin \theta \\
0 \\
-\cos \theta
\end{array}\right]
$$

where $\theta$ measures the angle from the $\hat{\mathbf{x}}$ axis. The force on the stylus dipole due to the solenoid can then be computed as the opposite of the magnetic force on a volume current, from [13]:

$$
\mathbf{F}_{\text {sol }}=-\int\left(\mathbf{J}_{\text {sol }} \times \mathbf{B}_{\text {sty }}(\mathbf{p})\right) \mathrm{d} V_{\text {sol }}
$$

\subsection{Force due to a Ferromagnetic Core}

For an electromagnet with an open air core, $\mathbf{F}_{\text {core }}$ will be zero. For an electromagnet with a ferromagnetic core with high magnetic susceptibility and low magnetic remanence, the core will amplify any magnetic fields present in the space that it occupies. The core will amplify the magnetic field produced by the solenoid, and it will also amplify the magnetic field produced by the stylus dipole. The former is a desirable effect, but the latter is not. Thus force due to the core $\mathbf{F}_{\text {core }}$ can be further decomposed into two distinct parts.

$$
\mathbf{F}_{\text {core }}=\mathbf{F}_{\text {core,sol }}+\mathbf{F}_{\text {core,sty }}
$$

\subsubsection{Core Force due to the Solenoid}

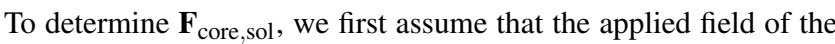
solenoid $\mathbf{H}_{\text {sol }}$ is uniform across a thin differential disk section of core. Thus we must only determine $\mathbf{H}_{\text {sol }}$ along the centerline of the core, which is given by the Biot-Savart law, and integrate that field over the disk:

$$
\mathbf{H}_{\text {sol }}(h)=\frac{1}{4 \pi} \int \frac{\mathbf{J}_{\text {sol }} \times \hat{\mathbf{y}}}{\left|h \hat{\mathbf{y}}-\mathbf{r}_{\text {sol }}\right|^{2}} \mathrm{~d} V_{\text {sol }}
$$

where $\mathbf{r}_{\text {sol }}$ is the vector from the origin to a differential volume element in the solenoid, $h$ is the distance from the origin to the center of a thin differential disk of core along $\hat{\mathbf{y}}$, and the "hat" operator indicates a unit-length vector [13]. The magnetization of a thin differential disk of core is then

$$
\mathbf{M}_{\text {core }, \text { sol }}(h)=\frac{1}{N} \mathbf{H}_{\text {sol }}(h)
$$

where $N$ is the demagnetization factor of the cylindrical core, which is a function of its geometry. This magnetization represents a bound current in the core, which is a collection of tiny current loops existing on the surface of an object that, from a macroscopic viewpoint, appear to be one current loop around the entire object. The bound current vector is:

$$
\mathbf{C}_{\mathrm{b}}(h)=\left|\mathbf{M}_{\mathrm{core}, \mathrm{sol}}(h)\right|\left[\begin{array}{c}
-\sin \theta \\
0 \\
-\cos \theta
\end{array}\right]
$$

We can use (6) to determine the field of the dipole at each point on the cylindrical surface of the core (i.e., not including the ends), where $\mathbf{p}$ is now the vector from the dipole to the differential surface element $\mathrm{d} A_{\text {core }}$. The force applied to the stylus due to the solenoid's magnetization of the core is then given by:

$$
\mathbf{F}_{\text {core }, \text { sol }}=\int\left(\mathbf{C}_{\mathrm{b}}(h) \times \mathbf{B}_{\text {sty }}(\mathbf{p})\right) \mathrm{d} A_{\text {core }}
$$

\subsubsection{Core Force due to the Stylus}

Finally, to determine $\mathbf{F}_{\text {core,sty }}$, we again use (6) to determine the stylus dipole's field at each differential volume element in the core $\mathrm{d} V_{\text {core }}$. Similar to (11), we then find a dipole moment for each differential volume element of the core $\mathrm{d} V_{\text {core }}$ :

$$
\mathbf{M}_{\text {core }, \text { sty }}(\mathbf{p})=\mathbb{N}^{-1} \mathbf{H}_{\text {sty }}(\mathbf{p})
$$

where $\mathbb{N}$ is now a demagnetization matrix and $\mathbf{H}_{\text {sty }}(\mathbf{p})=$ $\mathbf{B}_{\text {sty }}(\mathbf{p}) / \mu_{0}$. The force applied to the stylus due to the stylus' own magnetization of the core is then calculated by integrating the equation for the force between two dipoles throughout the volume of the core:

$$
\begin{aligned}
\mathbf{F}_{\text {core }, \text { sty }}= & \int \frac{3 \mu_{0}}{4 \pi|\mathbf{p}|^{4}}\left[\mathbf{m}_{\text {sty }} \hat{\mathbf{p}}^{T}+\hat{\mathbf{p}} \mathbf{m}_{\text {sty }}^{T}\right. \\
& \left.+\left(\hat{\mathbf{p}}^{T} \mathbf{m}_{\text {sty }}\right)\left(\mathbb{I}-5 \hat{\mathbf{p}} \hat{\mathbf{p}}^{T}\right)\right] \mathbf{M}_{\text {core }, \text { sty }}(\mathbf{p}) \mathrm{d} V_{\text {core }}
\end{aligned}
$$




\subsection{Model Validation with Experimental Prototype}

To understand the capabilities of our model, we compare the model with an FEA simulation in Ansoft Maxwell, using the parameters chosen to match our actual experimental interface. All volume and surface integrals in the model are evaluated numerically using MATLAB's triplequad and doublequad functions.

For our experimental setup, we chose an electromagnet with geometry of $R=25.4 \mathrm{~mm}, G=3.2 \mathrm{~mm}, W=14.3 \mathrm{~mm}, H=12.6 \mathrm{~mm}$. We chose a square-cross-section wire in order to get a tight packing, and we arbitrarily chose a 16-gauge wire. To power the electromagnet, we use an Advanced Motion Controls analog servo drive (model S16A8) with a maximum continuous current of $8 \mathrm{~A}$. We use an Advanced Motion Controls power supply (model PS2X3W24) capable of $300 \mathrm{~W}$ of output power. The resistance of our electromagnet is $2.9 \Omega$. At $1000 \mathrm{~Hz}$, the measured inductance of the electromagnet is 5.98 and $6.09 \mathrm{mH}$, and thus the electrical time constant is 2.06 and $2.10 \mathrm{~ms}$ for the air core and ferromagnetic core electromagnet, respectively. For the stylus, we chose an $11 \mathrm{~mm} \times 11 \mathrm{~mm}$ cylindrical grade-N52 NdFeB permanent magnet, with a dipole moment $\left|\mathbf{m}_{\text {sty }}\right|=1.26 \mathrm{~A} \cdot \mathrm{m}^{2}$.

From Fig. 8 it is clear that the FEA and model results follow the same trends, except in the lateral force $F_{x}$ when using a ferromagnetic core. The difference becomes larger as the stylus gets closer to the electromagnet, and is likely due to unmodeled edge effects in our model (e.g., the assumption that the field applied across a given differential disk of the core is constant). From this validation, we conclude that the analytical model developed here is sufficient to use in analytical design evaluation and optimization, with the understanding that any final design should be evaluated by FEA to obtain a more accurate model.

\section{Design Considerations}

When we consider Fig. 8, we see that the vertical forces generated by the electromagnet with the air core are maximized when the stylus is close to the electromagnet, and decay with distance, which is intuitive. In contrast, the forces generated by the electromagnet with the ferromagnetic core peak at a finite distance $(D \approx 17 \mathrm{~mm})$, and then decay as the stylus gets closer, due to the attractive force component between the stylus magnet and the core. We also see a point at which the force on stylus becomes zero $(D \approx 12 \mathrm{~mm})$, and at all distances closer the stylus would just be pulled into the core; it would be advisable to add a mechanical stop to not allow the stylus magnet to get any closer. We also see that significantly larger forces can be generated with the electromagnet with the ferromagnetic core. Although the numerical values are specific to our system, the trends that we observe will apply more generally.

It may seem intuitive that a ferromagnetic core will always result in a more powerful magnetic interface (i.e., larger $F_{y}$ ) than the same solenoid with an air core, but we find this intuition incorrect. Figure 9 shows the maximum force achievable with both core types, and the vertical position at which the maximum force occurs, for a given electromagnet design and only varying the dipole moment of the stylus magnet, $\mathbf{m}_{\text {sty }}$. For the air core, we observe a linear relationship between peak force and stylus-magnet strength as expected, with the location of peak force not changing. We also observe that as the stylus magnet gets larger, the benefit of the ferromagnetic core diminishes until a point at which the core actually serves to weaken the underlying solenoid rather than strengthen it, and that the location of the peak force moves away from the electromagnet. From the results of Fig. 8 and Fig. 9, it seems clear that the size/strength of the stylus magnet, as well as the desired location of the virtual wall with respect to the electromagnet, must both be considered when deciding if ferromagnetic-core or air-core electromagnets should be used in the design of a given untethered magnetic interface.
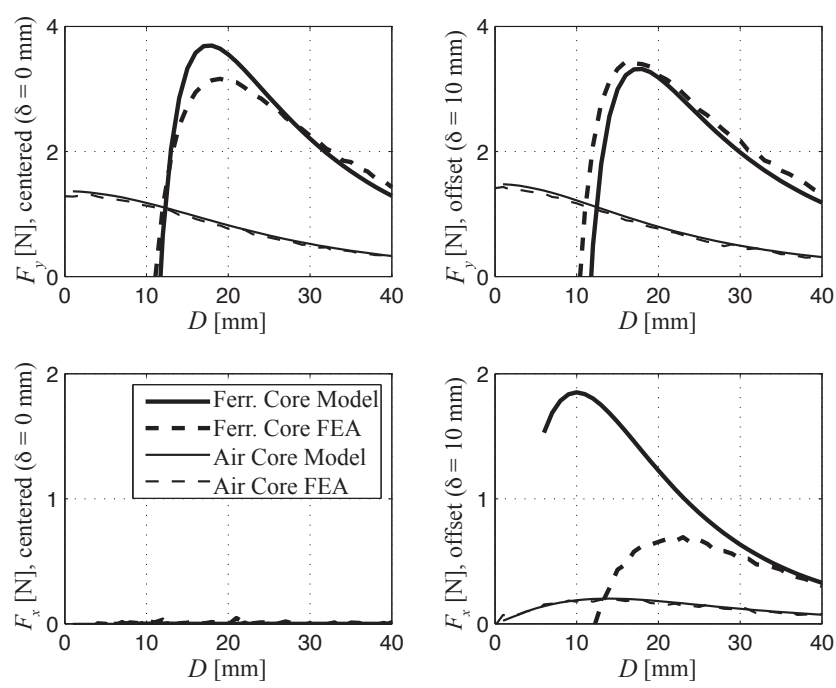

Figure 8: Magnetic-interface model compared to FEA results for both ferromagnetic-core and air-core electromagnets. The top row shows the (desirable) vertical repulsive force on the stylus, and the bottom row shows the (parasitic) lateral force on the stylus. The left column shows the case when the stylus is perfectly aligned with the axis of the electromagnet $(\delta=0)$, and we see that there is no lateral force on the stylus. The right column shows the case where the stylus is offset from the electromagnet's axis by $\delta=10 \mathrm{~mm}$, and we see there are significant lateral forces on the stylus (which are only partly captured by the model in the case of the ferromagnetic core).
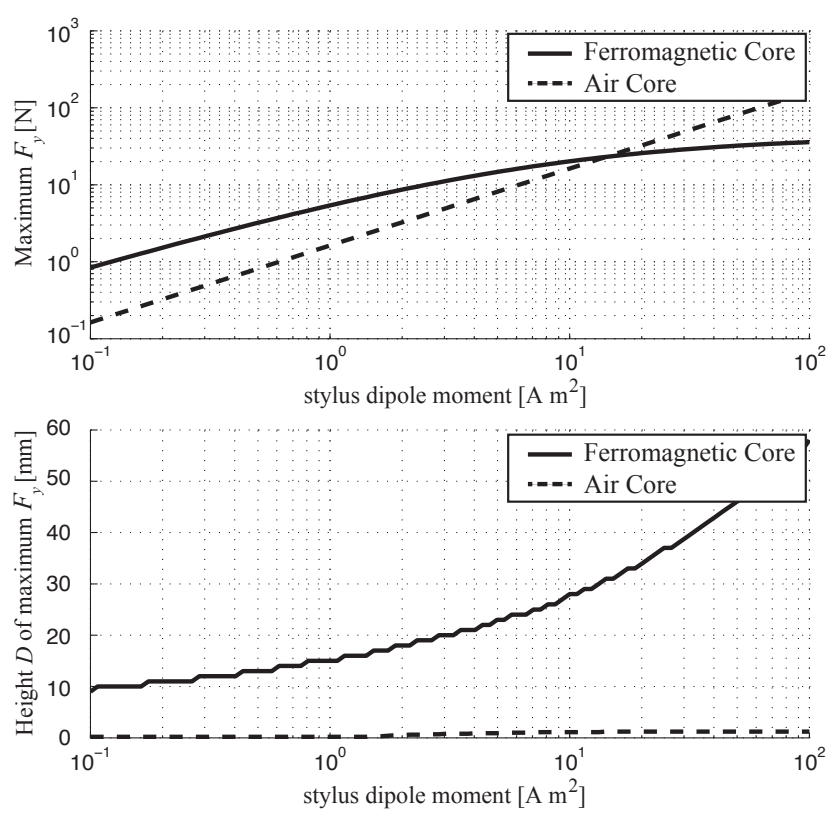

Figure 9: The maximum vertical force achievable with our electromagnet with a ferromagnetic core and an air core for varying stylus dipole moment $\mathbf{m}_{\text {sty }}$, and the location above the electromagnet at which the maximum force can be generated. A ferromagnetic core will enable more force for smaller stylus magnets, and an air core will enable more force for larger stylus magnets. With an air core, the peak force always occurs close to the electromagnet's surface, and with a ferromagnetic core, as the strength of the stylus magnet increases, the peak force moves farther away from the electromagnet. 


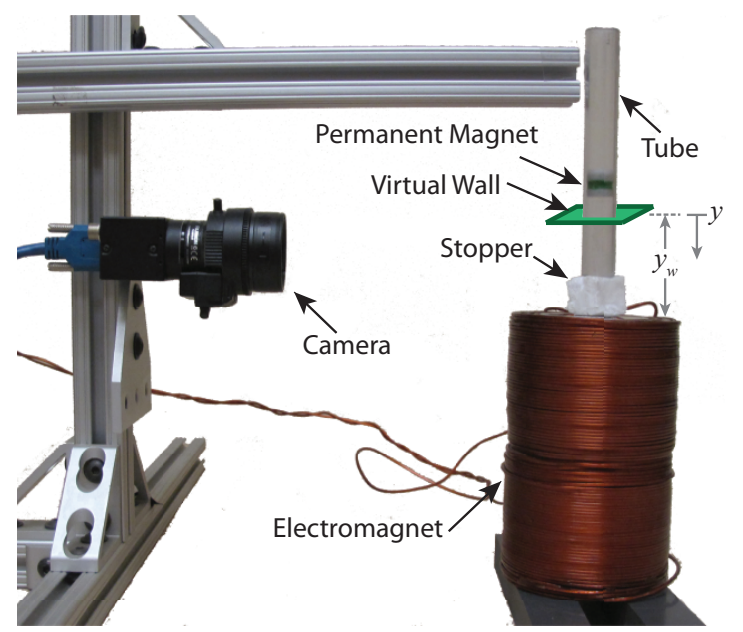

Figure 10: Experimental setup for virtual-wall stability experiment. A permanent magnet moves vertically in 1-DOF in a vertical tube. A camera, tracking a green dot painted on the permanent magnet, provides position feedback. Virtual walls are rendered at a variety of heights above the electromagnet, at two different sampling rates, and the stiffness at which instability is observed is recorded.

\section{EXPERIMENTS}

In the implementation of magnetic force, we recall that $F_{\text {sol }}$ and $F_{\text {core,sol }}$ scale linearly with the current applied to the solenoid, but $F_{\text {core,sty }}$ is independent of current. We therefore generate a lookup array for the sum of the two linear terms $F_{\text {sol }}$ and $F_{\text {core,sol }}$ with $1 \mathrm{~A}$ of applied current, and a separate lookup array for $F_{\text {core,sty }}$, at each height $D$ with a resolution of $1 \mathrm{~mm}$, using the values from the FEA model in Fig. 8. In real-time, we subtract $F_{\text {core,sty }}$ from the desired force and solve for the current required to achieve remaining force.

To explore the effects of virtual-wall location $y_{w}$, spring constant $K_{w}$, sampling time $T$, and air core vs. ferromagnetic core on stability, we conducted the following experiment (Fig. 10): The electromagnet is placed $160 \mathrm{~mm}$ away from a camera lens. A $15 \mathrm{~mm}$ styrofoam cube is fixed to the surface of the electromagnet, on top of which a $120 \mathrm{~mm}$ long, $12.7 \mathrm{~mm}$ inner-diameter plastic tube is secured. The permanent magnet described previously was fitted within a Delrin sleeve to bring the diameter of the cylindrical permanent magnet to just less than $12.7 \mathrm{~mm}$. The camera tracks the permanent magnet in a region of interest that is the dimensions of the tube. 10 pixels on the camera corresponds to $0.801 \mathrm{~mm}$ of travel along the tube. Although the tube creates friction in the system that is not modeled in the stability analysis, it allows the magnet to truly travel in only 1-DOF along the axis of the electromagnet. The excess friction present in the experiment will certainly affect the stability results, but the trends that are shown should generalize. The magnetic field of the electromagnet is controlled open-loop and updated given the position of the permanent magnet from the camera.

We ran four independent stability experiments in which we consider the four combinations of our solenoid with a ferromagnetic core and with an air core, and with sampling rates of $100 \mathrm{~Hz}$ and $50 \mathrm{~Hz}$, significantly slower than the electrical time constants of the magnet. For each automated experiment, we spanned a set of wall locations $y_{w}$ from $30 \mathrm{~mm}$ to $50 \mathrm{~mm}$, where $y_{w}$ is now the distance between the surface of the electromagnet and the virtual wall. At each $y_{w}$, we determined the critical virtual-wall spring constant $K_{w, \text { crit }}$ such that $K_{w}<K_{w, \text { crit }}$ resulted in a stable interaction and $K_{w} \geq K_{w, \text { crit }}$ resulted in an unstable interaction. This critical spring constant was found using a binary search through a range of spring constants $0<K_{w}<50 \mathrm{~N} / \mathrm{m}$ with a resolution of $0.1 \mathrm{~N} / \mathrm{m}$. In pilot studies we experimentally determined $50 \mathrm{~N} / \mathrm{m}$ was unstable for
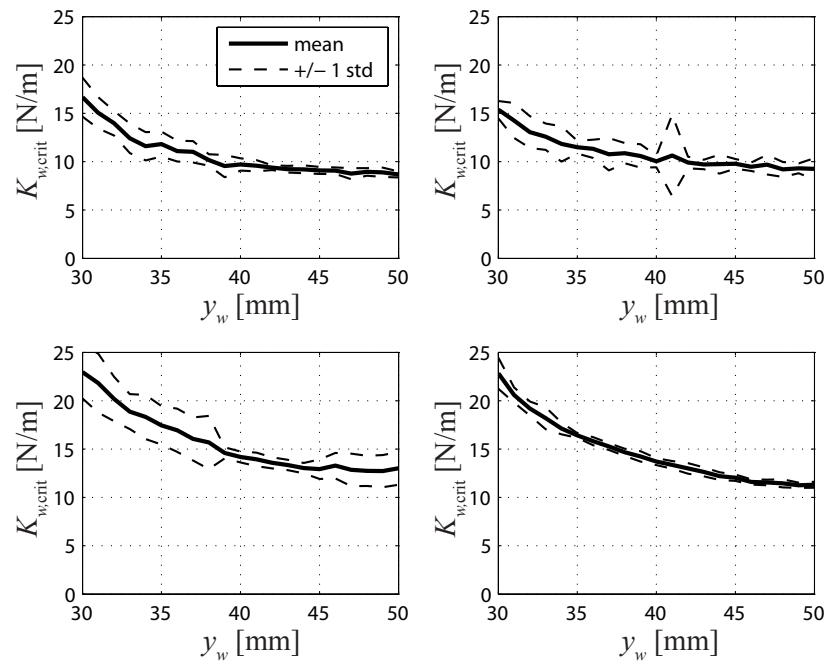

Figure 11: Results of virtual-wall stability experiment. The top row shows results the ferromagnetic core, and the bottom row shows results with the air core. The left column shows an update rate of $100 \mathrm{~Hz}$, and the right column shows an update rate of $50 \mathrm{~Hz}$. Mean and standard deviations for the critical virtual-wall stiffness is shown for 14 independent trials at each location.

all wall locations in our range. To determine whether a given $K_{w}$ resulted in a stable or unstable interaction, we first raised the permanent magnet $10 \mathrm{~mm}$ above the wall height using a PID position controller and held this position for 1 second. We then dropped the permanent magnet (by turning off the electromagnet) into the wall and tracked the permanent magnet as it oscillated about an equilibrium point for 3 seconds. If the permanent magnet spent $98 \%$ of the last second inside the wall, we determined this to be a stable interaction, otherwise we deemed the interaction unstable. This choice is conservative in that it demands significant damping to be present to declare stability, but it will offset the fact that our tube has unmodeled friction. We collected 14 passes of data at every $y_{w}$ for each experiment. The critical wall spring constant $K_{w, \text { crit }}$ is plotted against wall height $y_{w}$ for all four experiments in Fig. 11.

We observe that, as expected, increasing the height of the virtual wall above the electromagnet lowers the critical spring constant between stability and instability. The critical wall spring constants $K_{w, \text { crit }}$ are quite low even for soft virtual fixtures, however a more powerful system will generate steeper force curves and thus larger $K_{w, \text { crit }}$. We also observe that the stability is quite insensitive to the update rate, with very little difference between the $100 \mathrm{~Hz}$ and $50 \mathrm{~Hz}$ experiments. It appears that the inherent stability properties of the magnetic field between updates may supersede the effect due to the sampling rate- the effect that typically dominates consideration of stability in traditional virtual walls. In fact, increasing the sampling time $T$ will render previously stable interactions even more dissipative, and previously unstable interactions even more energetic, but the interactions on the verge of instability will remain on the verge of instability regardless of the sampling time. Finally, we observe that a stiffer virtual wall can be generated with the air-core electromagnet than with the ferromagnetic-core electromagnet. This result is quite counterintuitive, since it would be reasonable to assume that adding a ferromagnetic core would act to amplify the field of the solenoid (i.e., the air-core electromagnet), which would enable higher forces and thus higher stiffness. The additional eddy-current damping present with the ferromagnetic core would additionally act to stabilize the ferromagnetic-core experiments. Possible explanations for this unintuitive result are that 


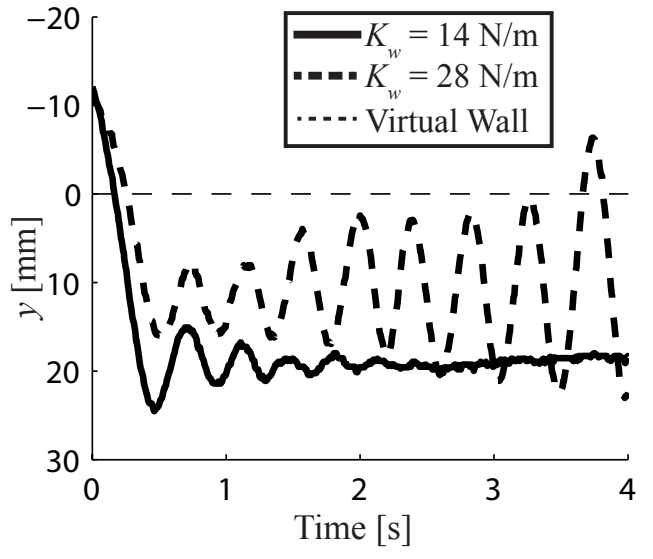

Figure 12: Haptic interaction with the experimental setup of Fig. 1. The virtual wall is rendered at $100 \mathrm{~Hz}$ by the air-core electromagnet at $y_{w}=40 \mathrm{~mm}$ at two stiffness values, chosen based on the results of Fig. 11. The interaction with the stiffer wall is unstable, and the interaction with the less-stiff wall appears stable but underdamped.

the attractive force between the stylus magnet and ferromagnetic core has the effect of mitigating the higher repulsive force curve expected with the ferromagnetic core, and that modeling error of the force is contributing to instability (see Fig. 8). More analysis and well-controlled experiments are required to fully describe the stability of untethered magnetic interfaces, but the experimental results shown in Fig. 11 are clear evidence that untethered magnetic interfaces exhibit stability properties that are significantly different from traditional haptic interfaces, and are even different from preconceived assumptions related to magnetic interfaces.

To see how the stability results in Fig. 11 extend to haptic interactions, we conclude with a set of experiments in which a human user interacts with the untethered magnetic interface shown in Fig. 1. Figure 12 shows experimental data for two examples of a user using the untethered stylus to interact with the virtual wall: one stable and one unstable. In these examples, we use the electromagnet with an air core and a sampling rate of $100 \mathrm{~Hz}$. The virtual wall was placed at $y_{w}=40 \mathrm{~mm}$ above the surface of the electromagnet. The expected spring constant to be just on the verge of instability is $K_{w, \text { crit }}=14 \mathrm{~N} / \mathrm{m}$ (see Fig. 11 ). This $K_{w}$ resulted in a stable but underdamped interaction with the virtual wall, supporting the stylus weight at $y=20 \mathrm{~mm}$. Doubling this critical spring constant to $K_{w}=28 \mathrm{~N} / \mathrm{m}$, resulted in an interaction that is clearly unstable. In both experiments, the stylus was held approximately $10 \mathrm{~mm}$ above the virtual wall before applying an approximately constant downward force by the hand. We find qualitatively that the $K_{w}$ stability values predicted in Fig. 11 always resulted in a stable interaction with the haptic stylus. This could be due in part to the additional inertia of the stylus compared to the permanent magnet alone $(22 \mathrm{~g}$ vs. $10 \mathrm{~g}$ ), and in part due to the damping in the human hand being larger than that in the tube. Regardless, it appears that the results from the automated hands-free experiment of Fig. 11 are indicative of the actual stability during haptic interaction. The low stiffnesses rendered in this experiment are evidence that generating stiff walls with an untethered device is a major design challenge.

\section{Conclusion}

We have presented an analysis of the factors affecting the performance, workspace, and stability of untethered magnetic haptic interfaces, with the goal of informing the design of future 2D and $3 \mathrm{D}$ devices. We developed a simple analytical model for a 1-DOF magnetic interface, to be used for design and optimization of mag- netic interfaces. We showed that even though untethered magnetic interfaces have little to no damping, they still exhibit inherent stability properties due to the continuous change in force between updates. We provided evidence that stability is insensitive to sampling rate, which deviates significantly from traditional haptic interfaces. Although a ferromagnetic core often enables increased forces to be rendered at distances farther from the electromagnet, we showed that there are cases in which adding a ferromagnetic core actually reduces the available magnetic force that can be rendered. Finally, we provided experimental evidence of a magnetic interface in which stiffer virtual walls can be rendered at a given distance from the electromagnet with an air core than with a ferromagnetic core.

\section{ACKNOWLEDGEMENTS}

This material is based upon work supported by the National Science Foundation under Grant No. 0654414.

\section{REFERENCES}

[1] J. J. Abbott and A. M. Okamura. Effects of position quantization and sampling rate on virtual-wall passivity. IEEE Trans. Robotics, 21(5):952-964, 2005

[2] R. J. Adams and B. Hannaford. Stable haptic interaction with virtual environments. IEEE Trans. Robotics and Automation, 15(3):465-474, 1999.

[3] P. Berkelman, S. Bozlee, and M. Miyasaka. Interactive dynamic simulations with co-located maglev haptic and $3 \mathrm{~d}$ graphic display. In Int. Conf. Advances in Computer-Human Interactions, pages 324 329, 2013.

[4] P. Berkelman and M. Dzadovsky. Extending the motion ranges of magnetic levitation for haptic interaction. In World Haptics, pages 517-522, 2009.

[5] P. J. Berkelman and R. L. Hollis. Lorentz magnetic levitation for haptic interaction: Device design, performance, and integration with physical simulations. Int. J. Robotics Research, 19(7):644-667, 2000.

[6] J. E. Colgate and J. M. Brown. Factors affecting the Z-Width of a haptic display. In Proceedings of the 1994 IEEE International Conference on Robotics and Automation, pages 3205-3210. IEEE Comput. Soc. Press, 1994.

[7] J. E. Colgate and G. Schenkel. Passivity of a class of sampled-data systems: Application to haptic interfaces. J. Robotic Systems, 14(2):3747, 1997.

[8] S. de Jong. Developing the cyclotactor. In Int. Conf. New Interfaces for Musical Expression, pages 163-164, 2009.

[9] S. de Jong. Presenting the cyclotactor project. In Int. Conf. Tangible, Embedded and Embodied Interaction, pages 319-320, 2010.

[10] N. Diolaiti, G. Niemeyer, F. Barbagli, and J. K. Salisbury, Jr. Stability of haptic rendering: Discretization, quantization, time-delay and coulomb effects. IEEE Trans. Robotics, 22(2):256-268, 2006.

[11] R. B. Gillespie and M. R. Cutkosky. Stable user-specific haptic rendering of the virtual wall. In ASME Dynamical Systems and Control Division, volume 58, pages 397-406, 1996.

[12] A. H. C. Gosline and V. Hayward. Eddy current brakes for haptic interfaces: Design, identification, and control. IEEE/ASME Trans. Mechatronics, 13(6):669-677, 2008.

[13] D. J. Griffiths. Introduction to Electrodynamics. Prentice Hall, fourth edition, 2013

[14] A. Z. Hajian and R. D. Howe. Identification of the mechanical impedance at the human finger tip. J. Biomechanical Engineering, 119:109-114, 1997.

[15] J. Hu, C.-Y. Chang, N. Tardella, J. Pratt, and J. English. Effectiveness of haptic feedback in open surgery simulation and training systems. Studies in health technology and informatics, 119:213-8, Jan. 2006.

[16] M. P. Kummer, J. J. Abbott, B. E. Kratochvil, R. Borer, A. Sengul, and B. J. Nelson. OctoMag: An electromagnetic system for 5-DOF wireless micromanipulation. IEEE Trans. Robotics, 26(6):1006-1017, 2010.

[17] S. E. Salcudean and T. D. Vlaar. On the emulation of stiff walls and static friction with a magnetically levitated input/output device. $J$. Dynamic Systems, Measurement, and Control, 119(1):127-132, 1997. 